\title{
The risk assessment by clinical background and cephalometry for obstructive sleep apnea with CPAP indication in Japanese
}

\author{
Shuhei Nozawa $^{1} \cdot$ Kazuhisa Urushihata $^{1}$ (D) $\cdot$ Ryosuke Machida $^{1} \cdot$ Masayuki Hanaoka $^{1}$
}

Received: 10 September 2020 / Accepted: 10 December 2020 / Published online: 5 January 2021

(c) The Author(s) 2021

\begin{abstract}
Obstructive sleep apnea (OSA) is a disease characterized by a brief period of cessation or marked decline in ventilation. The standard treatment is continuous positive airway pressure (CPAP). To determine the indication, full-night polysomnography (PSG) is required; however, it is a stressful examination. We verified the usefulness of pulse-oximetry at home as a predictor for CPAP indication before PSG. Simultaneously, the clinical background and cephalometry were also verified. Among 376 subjects who received PSG at our hospital from February 2005 to April 2020, 138 subjects received pulse-oximetry at home before PSG were enrolled retrospectively and performed logistic regression analysis on those with CPAP indication. Concurrently, 262 of 376 subjects received cephalometry were enrolled, and logistic regression analysis were performed using clinical background and cephalometry. A 3\% oxygen desaturation (3\% ODI) on the pulse-oximeter was a predictor for CPAP indication. The positive predictive value of $3 \%$ ODI $\geq 28.5$ events $/ \mathrm{h}$ was $73.3 \%$ and the negative predictive value was $62.5 \%$. Older age, higher body mass index (BMI), stronger lower jaw retraction (wider $\angle \mathrm{ANB}$ ), and longer distance from the anterior superior tip of the hyoid bone to the mandibular plane (MP-H) on cephalometry were also independent predictors. The positive predictive value when both $\mathrm{BMI} \geq 26.0 \mathrm{~kg} / \mathrm{m}^{2}$ and $\mathrm{MP}-\mathrm{H} \geq 17.5 \mathrm{~mm}$ were present was $88.2 \%$ and the negative predictive value when both were absent was $71.3 \%$. The combination of clinical background and cephalometry would be a useful screening tool as well as a pulse-oximetry at home for CPAP indication.
\end{abstract}

Keywords Obstructive sleep apnea $\cdot$ Pulse-oximeter $\cdot$ Cephalometry $\cdot$ CPAP indication

\section{Introduction}

Obstructive sleep apnea (OSA), a common respiratory disease worldwide occurring from airway stenosis due to collapse of the upper airway during sleep, is estimated to affect approximately $14 \%$ of men and $5 \%$ of women [1]. Airway stenosis is caused largely by obesity or abnormal structure of the upper airway, causing cessation or marked decline of ventilation, called apnea and hypopnea. These, in turn, cause nocturnal hypoxia and hypercapnia leading to sympathetic activation, associated with a higher prevalence of arrhythmia

Supplementary Information The online version contains supplementary material available at https://doi.org/10.1007/s4110 5-020-00301-w.

Kazuhisa Urushihata

ichiju@shinshu-u.ac.jp

1 First Department of Internal Medicine, Shinshu University School of Medicine, 3-1-1 Asahi, Matsumoto, Japan and hypertension [2]. The treatment of OSA with severe symptoms depends on the number of apnea and hypopnea per hour represented as apnea hypopnea index (AHI) measured by full-night polysomnography (PSG). Although the gold standard treatment is continuous positive airway pressure (CPAP), it requires expensive medical equipment and is not indicated unless patients have a certain level of severity. Marin et al. conducted a 10-year cohort study targeting healthy subjects $(n=264)$, simple snores $(n=377)$, patients with mild or moderate OSA $(5<\mathrm{AHI}<30$ events $/ \mathrm{h}, n=403)$, patients with severe OSA (AHI $>30$ events/h, $n=235$ ), and patients with OSA treated with CPAP $(n=372)$. They concluded that patients with severe OSA had a much greater mortality than other groups and that mortality could be lowered to the level of healthy subjects by CPAP [3]. On Japanese insurance, CPAP indication determined by full-night PSG is (1) having some severe symptoms of OSA, such as daytime sleepiness, headache when waking up, (2) AHI $\geq 20$ events/h on full-night PSG (3) observed sleep disruption and marked reduction in deep sleep stage on full-night PSG, 
which improves after CPAP. In subjects receiving full-night PSG at our hospital from February 2005 to April 2020, 223 of $376(59.3 \%)$ patients were diagnosed with OSA, which had an indication for CPAP and was treated; it was found that 153 of 376 (40.7\%) patients did not require CPAP. We believe that risk assessment using data other than full-night PSG could predict the CPAP indication in advance, and help to determine whether to perform full-night PSG.

One of the most famous and useful screening tools is the pulse-oximeter. Apnea and hypopnea are associated with a transient decline in oxygen saturation $\left(\mathrm{SpO}_{2}\right)$, which a pulse-oximeter can detect. Flemons et al. reported the high ability of the pulse-oximeter in detecting OSA [4] in their systematic review.

Other methods for estimating OSA and severity without overnight examination have been reported. First, age might be a candidate for estimation. Elderly people have a higher prevalence of OSA, with older adults having a more severe level of AHI [5, 6]. This may be because muscle function, related to upper airway patency declines in elderly people, leading to deterioration of OSA. Second, there may be gender differences with OSA being more common in men than in women. An epidemiological survey of gender ratio demonstrated the ratio (men: women) to be 2-3: 1 [7-9]. Although the cause is unknown, the role of sex hormones is considered. Cistulli et al. reported that testosterone exacerbates sleep-related dysfunction (SDB) [10], while Young et al. reported a prominent increase in OSA in women after menopause [11], indicating that progesterone could work defensively for OSA. Therefore, gender differences could be a predictor. Third, we evaluated daytime sleepiness using a questionnaire. Daytime sleepiness is a typical symptom of OSA, and the more severe the disease, the stronger the symptoms. Thus, evaluating daytime sleepiness might be a predictor of OSA severity. The Epworth Sleepiness Scale (ESS), developed in 1991 by an Australian physician, John W. Murray, is a widely used method for evaluating subjective daytime sleepiness [12]. This may contribute to the analysis and symptoms that are easy to apply. Fourth, obesity was represented by body mass index (BMI). Fat deposition around the upper airway causes airway stenosis, and obesity is an obvious factor of OSA. Sareli et al. reported the prevalence of OSA in a severely obese population as $93.6 \%$ in men and $73.5 \%$ in women [13]. Peppard et al. through a 4 -year cohort study reported a $10 \%$ weight gain resulting in a $32 \%$ increase in $\mathrm{AHI}$ and a six-fold increase in the probability of the transition rate from a moderate to severe level of OSA [14]. It was possible to estimate the severity from the BMI. Fifth, cephalometry was used to measure the upper airway structure. Patients with OSA have a distinctive long face, mandible retraction, lower hyoid bone, and longer soft palate, tending to induce SDB
[15-19]. As Japanese are less obese compared to Westerners [20], their OSA pathology can be closely related to upper airway abnormality. Sakakibara et al. analyzed the characteristics of the upper airway on cephalometry in Japanese patients with OSA and showed that they tended to have a longer distance from the posterior nasal spine to the tip of the soft palate (PNS-P) and distance from the point anterior-superior tip of the hyoid bone to the mandibular plane (MP-H) [21]. There are only a few reports on risk assessment combining these data for CPAP indication.

We verified the usefulness of the pulse-oximeter at home for estimating OSA with CPAP indication. At the same time, verification of whether the predictors obtained from clinical background and cephalometry could be a useful screening tool for CPAP indication was also performed, as reported here.

\section{Materials and methods}

\section{Patients}

We performed full-night PSG in 376 subjects at our hospital from February 2005 to April 2020. CPAP indication was judged by our sleep specialist physician according to Japanese insurance coverage, which included some severe symptoms of OSA, AHI $\geq 20$ events/h, and appearance of sleep disruption and marked reduction in deep sleep stage on fullnight PSG. The following two verifications were performed. Our study was approved by the research ethics committee of Shinshu University School of Medicine (Permission number: 4827). The study protocols were performed in accordance with the principles outlined in the Declaration of Helsinki of the World Medical Association.

Verification for pulse-oximetry at home: among 376 subjects, 138 subjects received an out-of-center sleep test (OCST) including pulse-oximeter at home for OSA screening within 3 months of full-night PSG. Patients were enrolled retrospectively (Fig. 1) and the usefulness of the pulse-oximeter at home for estimating CPAP indication was analyzed.

Verification for clinical background and cephalometry: among 376 subjects, 262 were evaluated for their upper airway structure using cephalometry. We retrospectively enrolled 262 subjects and divided them into those with CPAP indication and those without (Fig. 2). Multivariate analysis was performed on the predictors of CPAP indication.

\section{Japanese version of Epworth Sleepiness Scale (JESS)}

Subjects were surveyed on daytime sleepiness using a Japanese version of the ESS (JESS) [22], a self-administered 


\section{Subjects who were received full-night PSG at our hospital from February 2005 to April 2020 \\ $\mathrm{N}=376$}

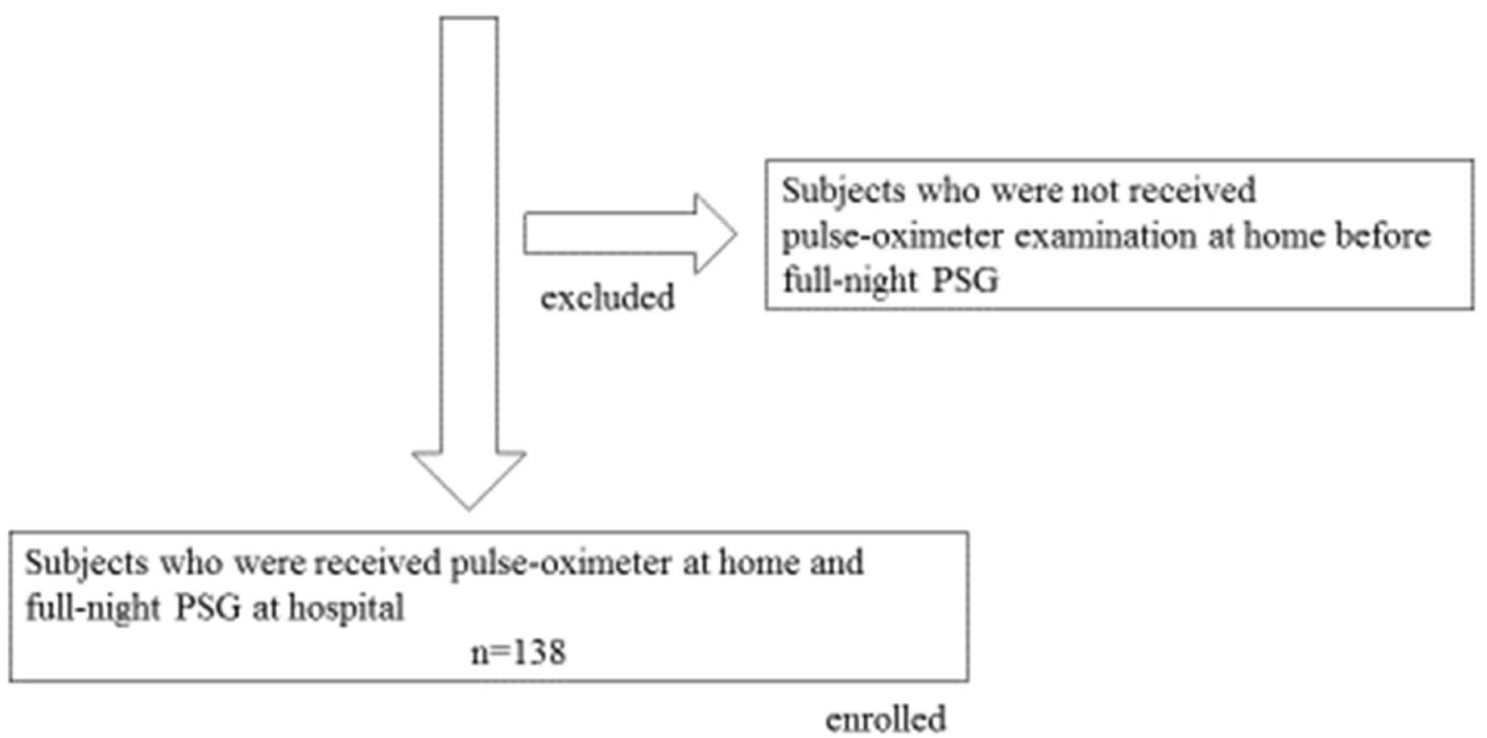

Fig. 1 Process of choosing patients in verification for pulse-oximeter at home. PSG polysomnography

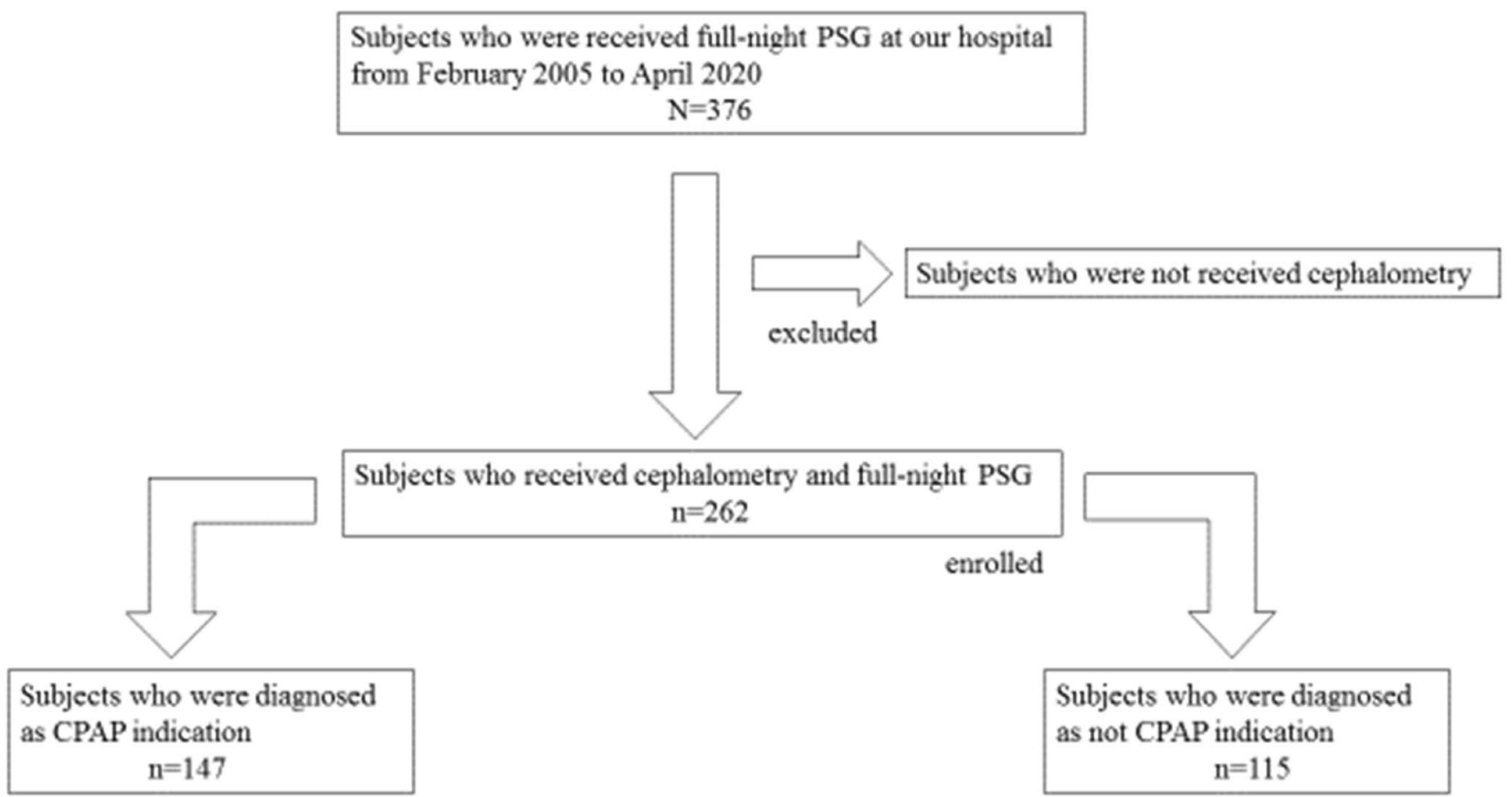

Fig. 2 Process of choosing patients in verification for clinical background and cephalometry. PSG polysomnography, CPAP contentious positive airway pressure 
questionnaire with eight questions, which is used to evaluate daytime sleepiness. Subjects were asked to rate, on a four-point scale (0-3), their usual chances of dozing off or falling asleep while engaged in eight different activities. The ESS score (the sum of eight item scores, 0-3) ranges from 0 to 24 , with a score $\geq 11$, defined as the propensity of sleep apnea syndrome.

\section{Full-night PSG examination}

The full-night PSG examination was undergone using Alice3 (Philips, Amsterdam, Nederland) and Embla N7000 (Embla, California, United States), which consisted of four-channel electroencephalogram (C4/A1,C3/A2,O2/ A1,O1/A2), two-channel electrooculogram, sub-mental and leg electromyogram, airflow (thermistor and pressure transducer), respiratory effort (chest and abdominal movement), $\mathrm{SpO}_{2}$, snoring, electrocardiogram and body position. OSA was diagnosed if patients had an AHI $\geq 5$ events/h and obstructive apnea dominant. AHI consisted of the total number of obstructive apnea (OA), central apnea (CA), mixed apnea (MA), and hypopnea and these respiratory events were scored according to the 'Alternative' rule of American Academy of Sleep Medicine [23]. OA is defined as airflow cessation with duration $>10$ s in the presence of thoracic and abdominal movements, while CA is the airflow cessation due to the absence of thoracic and abdominal movements, and MA is a mixture of OA and
CA. Hypopnea is defined as a reduction in flow of $\geq 50 \%$ with $>3 \%$ oxygen desaturation or arousal, in the presence of abdominal and thoracic movements. All PSG data were manually scored.

\section{Pulse-oximetry}

Pulse-oximetry was performed using a type III PM OCST (Smart Watch PMP-300E, Philips, Amsterdam, The Netherlands) at home before the full-night PSG. It measured nasal airflow (pressure transducer), respiratory effort (chest and abdominal movement), $\mathrm{SpO}_{2}$, snoring, electrocardiogram and body position. Mean $\mathrm{SpO}_{2}$, lowest $\mathrm{SpO}_{2}$, $3 \%$ oxygen desaturation (3\% ODI) and the percentage of time spent at $\mathrm{SpO}_{2}$ below 90\% (CT90) were calculated from nocturnal $\mathrm{SpO}_{2}$.

\section{Cephalometry}

The upper airway of the subjects was taken from the side area (Fig. 3) [24]. Skull base length (SN), upper jaw protrusion $(\angle \mathrm{SNA})$, lower jaw protrusion $(\angle \mathrm{SNB})$, lower jaw retraction $(\angle \mathrm{ANB})$, inferior airway space (IAS), PNS-P and MP-H reporting the significant differences against normal subjects $[18,25,26]$ were measured manually using electronic medical records system (EV Insite; PSP Inc, Tokyo, Japan).
Fig. 3 Cephalometric landmarks with line and area measurements [24]. $S$ sella, $N$ nasion, $A A$-point, deepest point of maxilla, $B B$-point, deepest point of mandible, $P$ palate point, $P N S$ posterior nasal spine, $H$ superior tip of hyoid bone, $S N$ skull base length, $\angle S N A$ upper jaw protrusion, $\angle S N B$ lower jaw protrusion, $\angle A N B$ lower jaw retraction, IAS inferior airway space, $P N S$ - $P$ distance from posterior nasal spine to tip of the soft palate, $M P-H$ distance from point anterior-superior tip of hyoid bone to mandibular plane

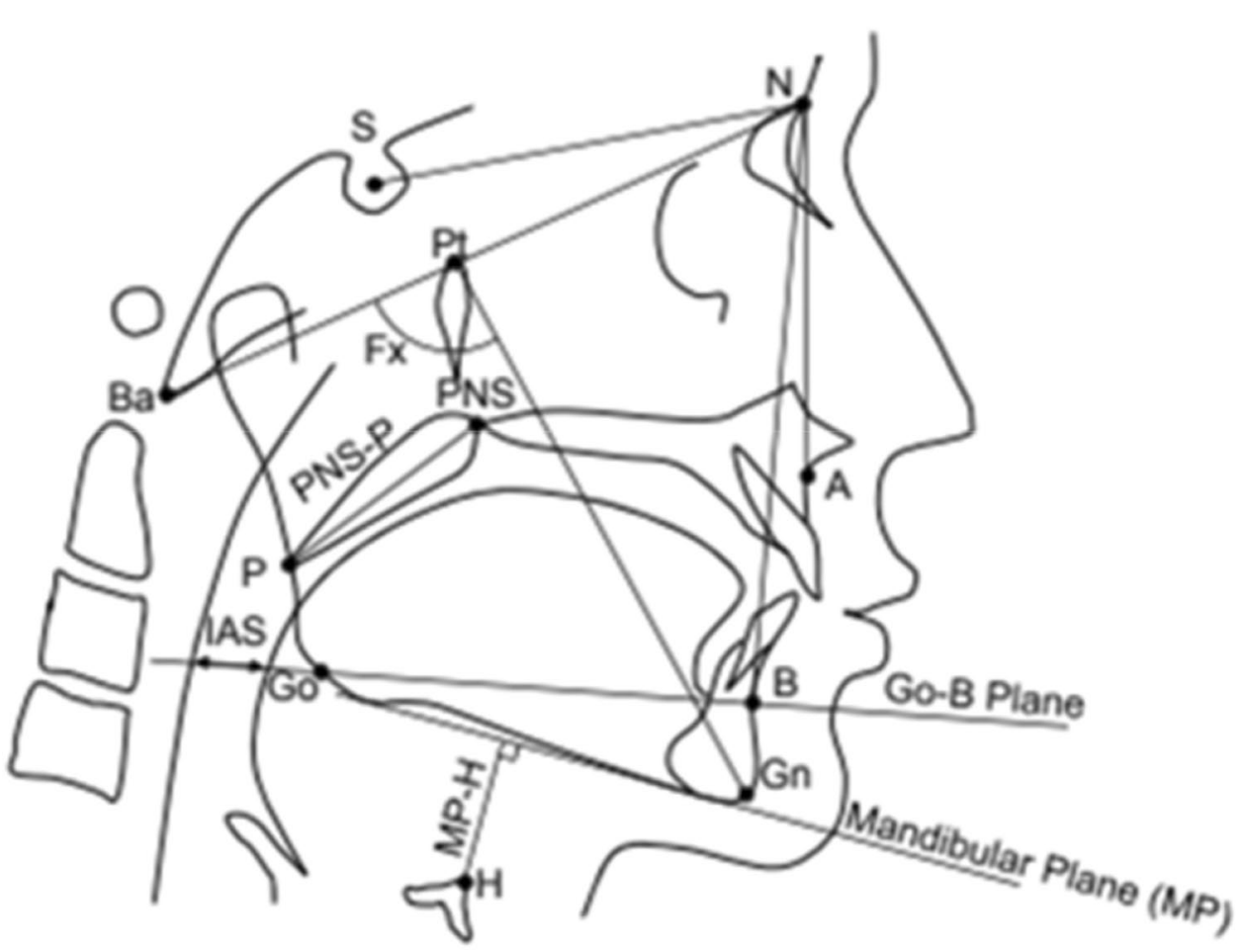




\section{Data analysis}

Statistical analyses were performed using SPSS Statistics (version 26, IBM, USA, Ltd). Measurement data are expressed as mean \pm standard deviation (SD). Categorical variables were compared using the Chi-square test. The comparison of variables between the two groups was first assessed by the Kolmogorov-Smirnov test to confirm whether they were normally distributed. Following this, we confirmed that they were equal or unequal in variance using the Levene test. If they were normal and equally distributed, Student's $t$ test was used, and if they were normal with unequal distribution, Welch's $t$ test was used. However, if they were not normally distributed, the MannWhiney $U$ test was used.

For verification of the pulse-oximetry at home, logistic regression analysis was performed by selecting CPAP indication as the dependent variable and 3\% ODI (events/h) was selected as the independent variable.

To verify the clinical background and cephalometry, logistic regression analysis was performed by selecting CPAP indication as the dependent variable and age, gender difference, BMI, JESS, $\angle \mathrm{ANB}$, and MP-H on cephalometry were selected as independent variables.
A correlation diagram was drawn using Spearman's correlation coefficient. A $p$ value of less than 0.05 was considered to indicate statistical significance.

\section{Results}

Verification for pulse-oximetry at home: 3\% ODI was a significant independent predictor of CPAP indication ( $p<0.001$; odds ratio [OR], 1.06; 95\% confidence interval [CI], 1.03-1.09). A positive correlation was observed between AHI and 3\% ODI (Fig. 4). The cutoff value of 3\% ODI for CPAP indication obtained from receiver operating characteristic (ROC) curve analysis (Fig. 5) was 28.5 events/h. The positive predictive value of $3 \%$ ODI $\geq 28.5$ events/h was $73.3 \%$ (66/90) and the negative predictive value was $62.5 \%(30 / 48)$ (Table 1$)$.

Verification of clinical background and cephalometry: clinical background and the results of full-night PSG of the two groups (CPAP indication vs. no CPAP indication) are shown in Table 2, and cephalometry is shown in Table 3. Patients with CPAP indication had statistically significant differences in gender, BMI, $\angle \mathrm{ANB}$, and MP-H on cephalometry. In terms of PSG, nocturnal oxygenation represented mean $\mathrm{SpO}_{2}$, lowest $\mathrm{SpO}_{2}, 3 \%$ ODI and CT90 was worse, and arousal and snoring index were higher in patients with

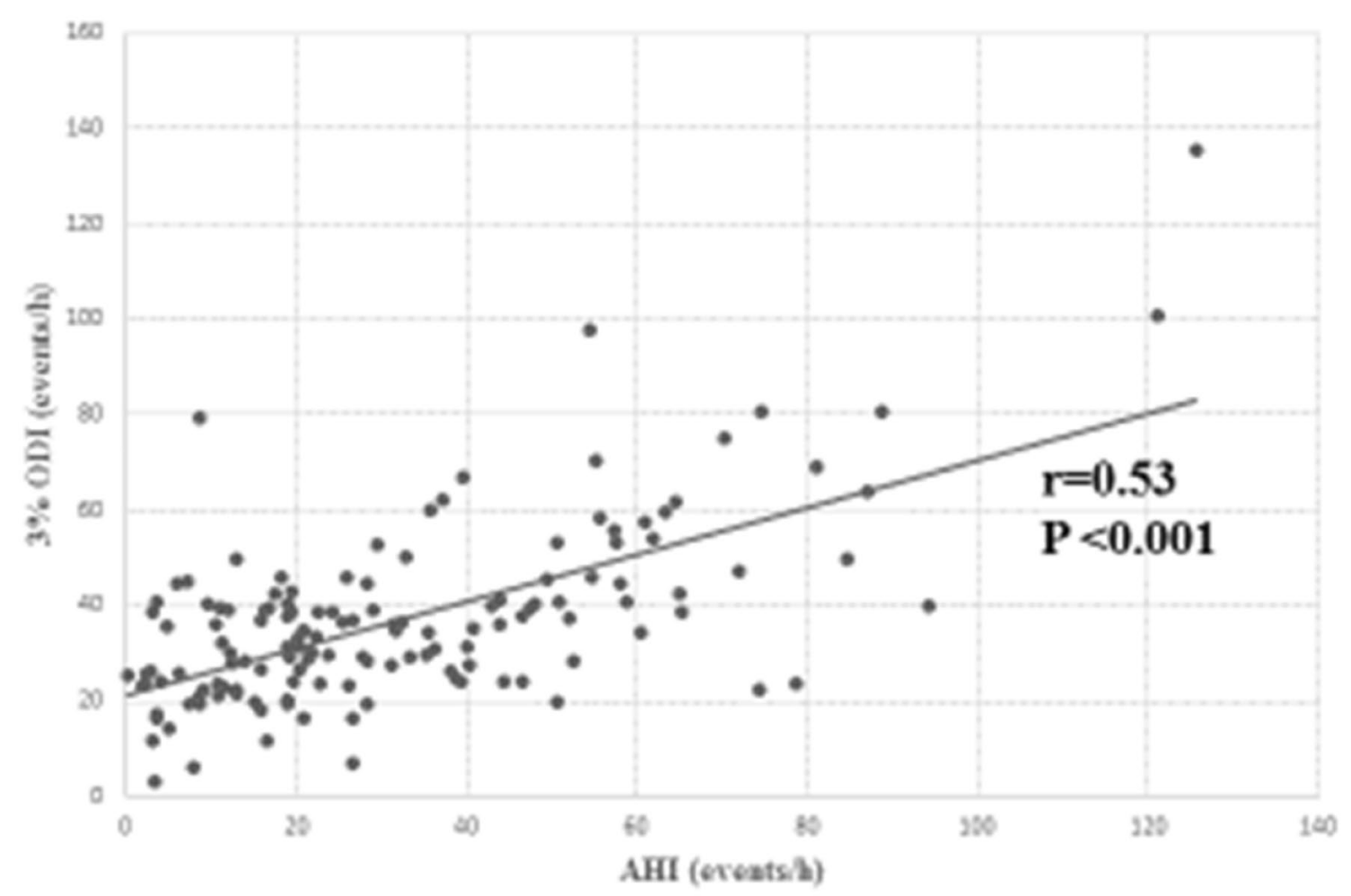

Fig. 4 Correlation diagram between AHI and 3\% ODI. AHI apnea hypopnea index, 3\% ODI 3\% oxygen desaturation index 
Fig. 5 ROC curve of $3 \%$ ODI for CPAP indication. ROC curve receiver operating characteristic curve, $3 \%$ ODI $3 \%$ oxygen desaturation index, CPAP contentious positive airway pressure

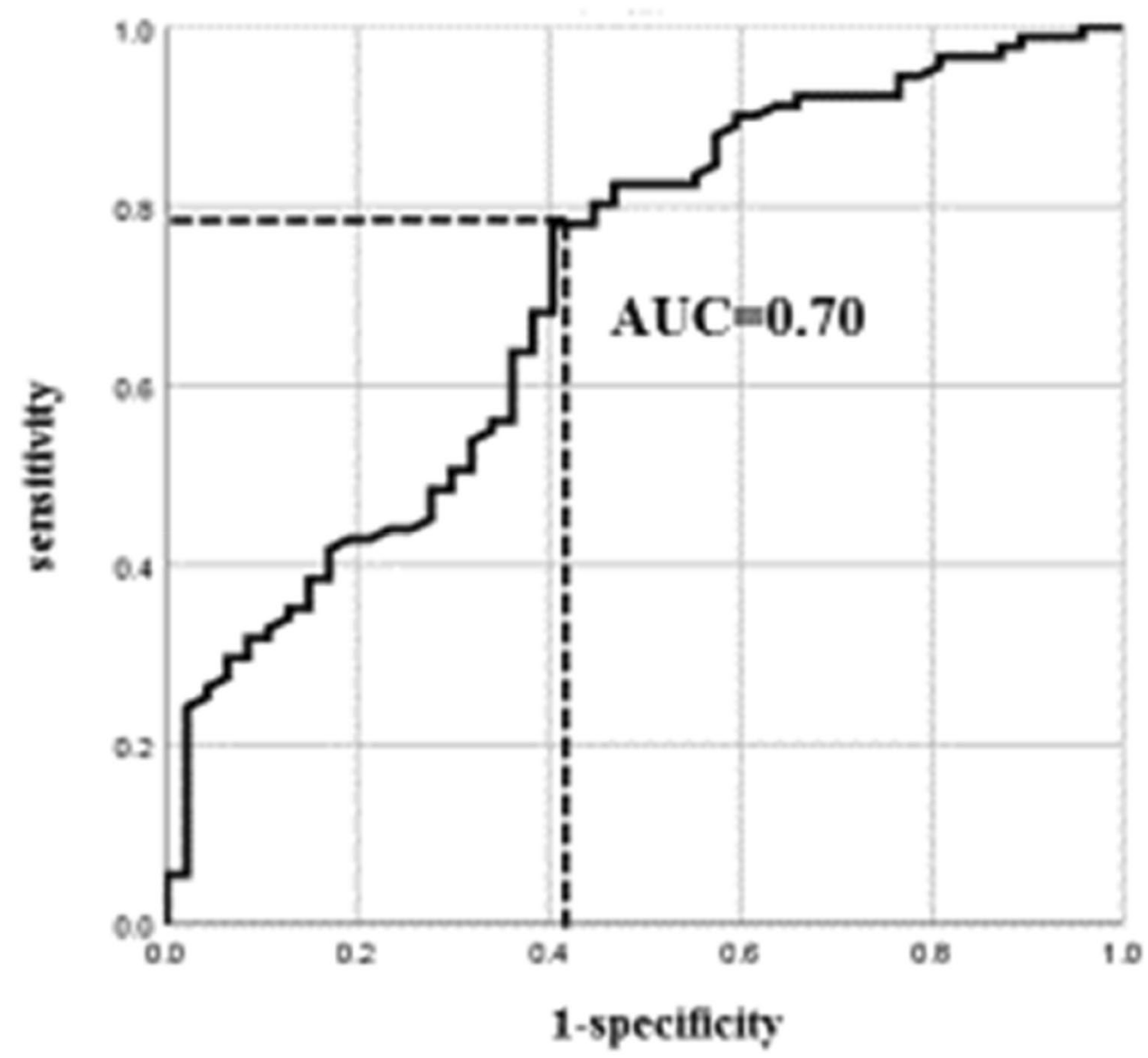

Table 1 Number of subjects with and without risks of $3 \%$ ODI $\geq 28.5$ events/h

\begin{tabular}{lllr}
\hline Variables & $\begin{array}{l}\text { CPAP indica- } \\
\text { tion }\end{array}$ & $\begin{array}{l}\text { Not CPAP } \\
\text { indication }\end{array}$ & Total \\
\hline $3 \%$ ODI $\geq 28.5$ events $/ \mathrm{h}$ & 66 & 24 & 90 \\
$3 \%$ ODI $<28.5$ events $/ \mathrm{h}$ & 18 & 30 & 48 \\
Total & 84 & 54 & 138 \\
\hline
\end{tabular}

CPAP contentious positive airway pressure, 3\% ODI 3\% oxygen desaturation index

CPAP indication compared to patients with no CPAP indication. The results of the logistic regression are shown in Table 4. Age, BMI, $\angle \mathrm{ANB}$, and MP-H were significant independent variables predicting CPAP indication. There was a positive correlation between $\mathrm{AHI}$ and $\mathrm{BMI}$, and $\angle \mathrm{ANB}$, and MP-H, although not in age (Fig. 6). The cutoff values were age $\geq 67.0$ years, $\mathrm{BMI} \geq 26.0 \mathrm{~kg} / \mathrm{m}^{2}, \angle \mathrm{ANB} \geq 4.4^{\circ}$, and $\mathrm{MP}-\mathrm{H} \geq 17.5 \mathrm{~mm}$, obtained from the receiver operating characteristic (ROC) curve (Fig. 7). When all four predictors were present, the positive predictive value was $100 \%(8 / 8)$ and when all predictors were absent, the negative predictive value was $82.5 \%$ (33/40) (Supplemental Table 1), demonstrating excellent screening function. However, it had few target patients, because few patients met all four predictors or did not meet any of four predictors, so we chose BMI and MP-H as two of the strong predictors. When both risks of $\mathrm{BMI} \geq 26.0 \mathrm{~kg} / \mathrm{m}^{2}$ and $\mathrm{MP}-\mathrm{H} \geq 17.5 \mathrm{~mm}$ were present, the positive predictive value was $88.1 \%(52 / 59)$ and when both risks were absent, the negative predictive value was $71.3 \%$ (62/87) (Table 5).

\section{Discussion}

Our present study demonstrated that pulse-oximetry at home could predict CPAP indication. Similarly, age, BMI, $\angle A N B$, and MP-H on cephalometry were also predictors of CPAP indication. A wider $\angle \mathrm{ANB}$ represents a more retracted lower jaw and a longer MP-H represents the lower tongue. Both findings are typical of OSA, indicating the possibility of upper airway narrowing. The two primary causes of OSA are obesity and abnormality of the upper airway structure. Therefore, evaluating these using BMI and cephalometry will be a useful screening tool for estimating the severity of OSA.

On the other hand, gender differences and daytime sleepiness evaluated using JESS were not predictors of CPAP 
Table 2 Clinical background and full-night polysomnography data between two groups

\begin{tabular}{|c|c|c|c|}
\hline Variables & CPAP indication & Not CPAP indication & $p$ value \\
\hline$n(\mathrm{M} / \mathrm{F})$ & $147(\mathrm{M}=110 / \mathrm{F}=37)$ & $115(\mathrm{M}=73 / \mathrm{F}=42)$ & 0.048 \\
\hline Age (years) & $60.8 \pm 14.4$ & $58.1 \pm 13.1$ & 0.050 \\
\hline $\operatorname{BMI}\left(\mathrm{kg} / \mathrm{m}^{2}\right)$ & $27.8 \pm 5.2$ & $25.3 \pm 4.2$ & $<0.001$ \\
\hline Smoking history (pack-years) & $24.3 \pm 29.2$ & $22.2 \pm 32.8$ & 0.71 \\
\hline JESS (point) & $6.0 \pm 4.4$ & $6.1 \pm 4.2$ & 0.71 \\
\hline AHI (events/h) & $47.0 \pm 18.4$ & $9.4 \pm 6.2$ & $<0.001$ \\
\hline TST (min) & $402.9 \pm 101.5$ & $410.6 \pm 84.5$ & 0.79 \\
\hline $\mathrm{SE}(\%)$ & $73.8 \pm 15.2$ & $72.9 \pm 14.1$ & 0.39 \\
\hline REM sleep time (\%) & $11.8 \pm 8.1$ & $14.3 \pm 6.5$ & 0.010 \\
\hline Stage N1 (\%) & $36.5 \pm 20.6$ & $24.0 \pm 13.6$ & $<0.001$ \\
\hline Stage N2 (\%) & $48.1 \pm 18.4$ & $53.7 \pm 13.8$ & 0.0010 \\
\hline Stage N3 (\%) & $3.8 \pm 5.0$ & $7.2 \pm 8.7$ & 0.022 \\
\hline Mean $\mathrm{SpO}_{2}(\%)$ & $92.3 \pm 2.6$ & $94.0 \pm 2.2$ & $<0.001$ \\
\hline Lowest $\mathrm{SpO}_{2}(\%)$ & $72.4 \pm 10.8$ & $83.2 \pm 6.6$ & $<0.001$ \\
\hline $3 \%$ ODI (events/h) & $42.8 \pm 19.8$ & $8.9 \pm 6.3$ & $<0.001$ \\
\hline СT90 (\%) & $19.7 \pm 18.5$ & $7.1 \pm 17.9$ & $<0.001$ \\
\hline Arousal index (events/h) & $42.5 \pm 29.9$ & $21.2 \pm 9.9$ & $<0.001$ \\
\hline Snoring index (events/h) & $67.9 \pm 60.9$ & $42.5 \pm 82.9$ & $<0.001$ \\
\hline
\end{tabular}

Data are expressed as mean \pm standard deviation $(\mathrm{SD})$

$C P A P$ contentious positive airway pressure, $M$ male, $F$ female, BMI body mass index, JESS Japanese version of Epworth Sleepiness Scale, AHI apnea hypopnea index, TST total sleep time, $S E$ sleep efficiency, TST time in bed (\%), REM sleep time rapid eye movement sleep time, 3\% ODI 3\% oxygen desaturation index, CT90 total sleep time with $\mathrm{SpO}_{2}$ under $90 \%$
Table 3 Cephalometry data between two groups

\begin{tabular}{lccc}
\hline Variables & CPAP indication & Not CPAP indication & $p$ value \\
\hline SN $(\mathrm{mm})$ & $73.2 \pm 5.9$ & $73.2 \pm 5.8$ & 0.99 \\
$\angle \mathrm{SNA}\left(^{\circ}\right)$ & $61.5 \pm 4.8$ & $60.7 \pm 5.4$ & 0.20 \\
$\angle \mathrm{SNB}\left(^{\circ}\right)$ & $57.4 \pm 4.6$ & $58.1 \pm 5.4$ & 0.46 \\
$\angle \mathrm{ANB}\left({ }^{\circ}\right)$ & $4.6 \pm 1.9$ & $3.7 \pm 1.8$ & 0.0010 \\
$\mathrm{IAS}(\mathrm{mm})$ & $9.8 \pm 3.8$ & $10.4 \pm 3.3$ & 0.12 \\
MP-H $(\mathrm{mm})$ & $18.1 \pm 6.9$ & $11.9 \pm 6.7$ & $<0.001$ \\
PNS-P $(\mathrm{mm})$ & $45.0 \pm 11.3$ & $48.0 \pm 10.7$ & 0.052 \\
\hline
\end{tabular}

$C P A P$ contentious positive airway pressure, $S N$ skull base length, $\angle S N A$ upper jaw protrusion, $\angle S N B$ lower jaw protrusion, $\angle A N B$ lower jaw retraction, IAS inferior airway space, $P N S-P$ distance from posterior nasal spine to tip of the soft palate, $M P-H$ distance from point anterior-superior tip of hyoid bone to mandibular plane

indication. Although differences in the prevalence of OSA between males and females have been reported for all ages [7-9], surveys for ages 65 or above showed smaller differences and menopause in females was correlated to this difference in prevalence $[27,28]$. The average age of females in this study was relatively high (average: $60.4 \pm 11.6$ years), which could explain the gender difference being not a predictor. Daytime sleepiness is a typical symptom that develops only to some extent [29]. Leticia et al. and Douglas et al. reported that ESS could detect severe levels of OSA but was inappropriate for mild and moderate levels and was
Table 4 Logistic regression analysis for CPAP indication

\begin{tabular}{llll}
\hline Variables & $p$ value & OR & $95 \% \mathrm{CI}$ \\
\hline Age (years) & 0.028 & 1.04 & $1.04-1.07$ \\
Gender difference & 0.69 & 1.18 & $0.52-2.70$ \\
BMI $\left(\mathrm{kg} / \mathrm{m}^{2}\right)$ & 0.0010 & 1.17 & $1.07-1.28$ \\
JESS (point) & 0.77 & 1.01 & $0.93-1.11$ \\
$\angle \mathrm{ANB}\left({ }^{\circ}\right)$ & 0.0070 & 1.30 & $1.08-1.58$ \\
$\mathrm{MP}-\mathrm{H}(\mathrm{mm})$ & 0.0020 & 1.10 & $1.04-1.16$ \\
\hline
\end{tabular}

$O R$ odds ratio, 95\% CI 95\% confidence interval, $B M I$ body mass index, JESS Japanese version of Epworth Sleepiness Scale, $\angle A N B$ lower jaw retraction, $M P-H$ distance from point anterior-superior tip of hyoid bone to mandibular plane

unsuitable for estimating the severity and could not replace full-night PSG [30, 31]. As in these previous reports, the evaluation of daytime sleepiness using JESS was not a predictor for estimating CPAP indication.

Although OSA is a common disease, it is difficult to diagnose as the symptoms even appear in people who do not have the disease. One of the typical symptoms was daytime sleepiness, but it can occur in other diseases, such as insomnia, central nervous system disorders, and mental illness. In a survey of the Japanese population, $14.9 \%$ experienced daytime sleepiness [32]. Another symptom of OSA is snoring, occurring in $70-95 \%$ of patients with OSA [33]. However, the prevalence of chronic snoring is $40 \%$ in adult men and 

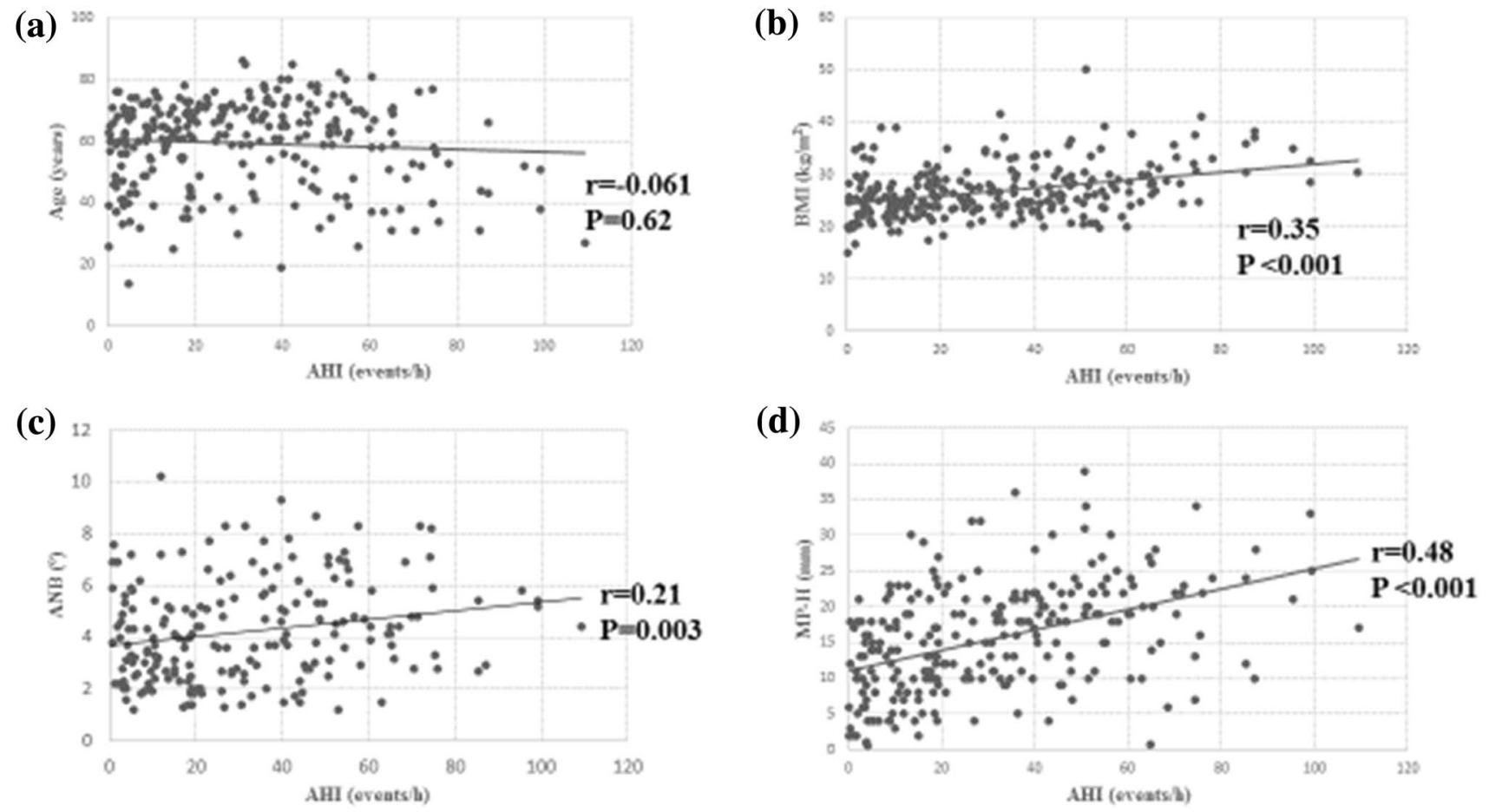

Fig. 6 Correlation diagram between AHI and age, BMI, $\angle A N B$, MP-H. a Correlation diagram between AHI and age. b Correlation diagram between $\mathrm{AHI}$ and BMI. c Correlation diagram between $\mathrm{AHI}$ and $\angle \mathrm{ANB}$. d Correlation diagram between AHI and MP-H.

$20 \%$ in adult women [34]. Considering these findings, it is difficult to estimate the AHI and predict CPAP indication from their symptoms. The surest method determining CPAP indication is full-night PSG, but it requires an overnight hospital stay and high cost. Although pulse-oximeter at home is a cheaper and simpler screening method, it also requires overnight examination. Risk assessment using clinical background and cephalometry could be a screening tool for the detection of patients with OSA who should be treated by CPAP without these disadvantages.

Our study had some limitations. First, the sample size was not large, because this study was conducted at a single institute with exploratory research. Second, risk assessment could be conducted only for patients with CPAP indication. However, patients with OSA patients who did not have CPAP indication at the time would have a feature risk of deterioration. Hence, regular follow-up and life guidance might be required in some cases after the diagnosis of OSA using full-night PSG even in patients who are unlikely to adapt to CPAP from this risk assessment.

In conclusion, pulse-oximetry at home could be a useful predictor for CPAP indication, as previously reported [4]. Similarly, the combination of BMI and MP-H on cephalometry could also be a strong predictor for CPAP indication without overnight examination. Risk assessment using

$A H I$ apnea hypopnea index, $B M I$ body mass index, $\angle A N B$ lower jaw retraction, $M P-H$ distance from point anterior-superior tip of hyoid bone to mandibular plane

it might reveal the requirement for full-night PSG, further reducing this stressful examination.

Acknowledgements We would like to thank Editage (www.edita ge.com) for English language editing.

\section{Compliance with ethical standards}

Conflict of interest For this study, we did not receive financial support from any organization.

Ethical committee permission The study was approved by the research ethics committee of Shinshu University School of Medicine (Permission number: 4827). The study protocols were performed in accordance with the principles outlined in the Declaration of Helsinki of the World Medical Association.

Research involving human and/or animals This study was conducted in accordance with the ethical principles described in the Declaration of Helsinki, the Japanese Ethical Guideline for Epidemiologic Research and all other applicable laws and regulations.

Open Access This article is licensed under a Creative Commons Attribution 4.0 International License, which permits use, sharing, adaptation, distribution and reproduction in any medium or format, as long as you give appropriate credit to the original author(s) and the source, provide a link to the Creative Commons licence, and indicate if changes were made. The images or other third party material in this article are included in the article's Creative Commons licence, unless indicated 


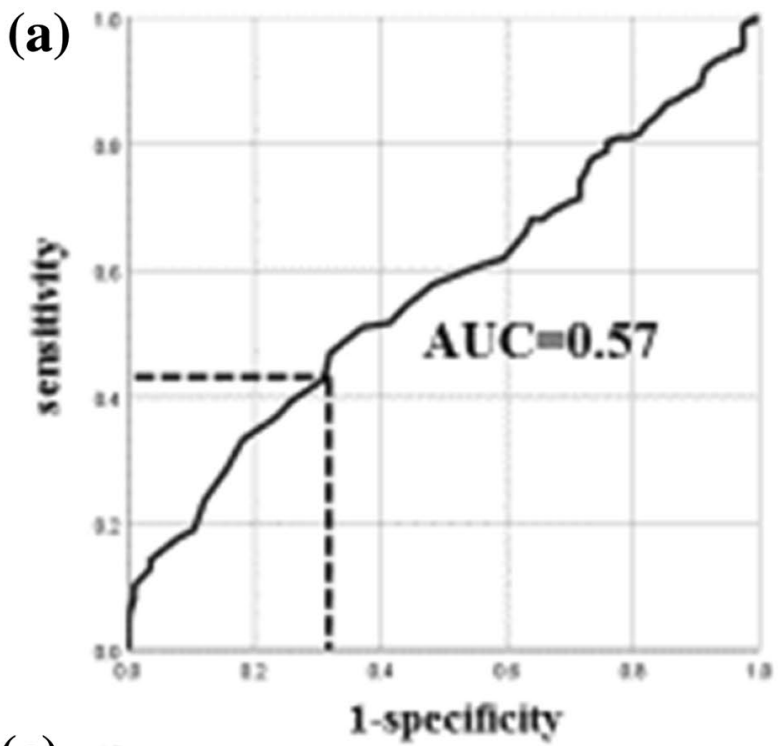

(c)

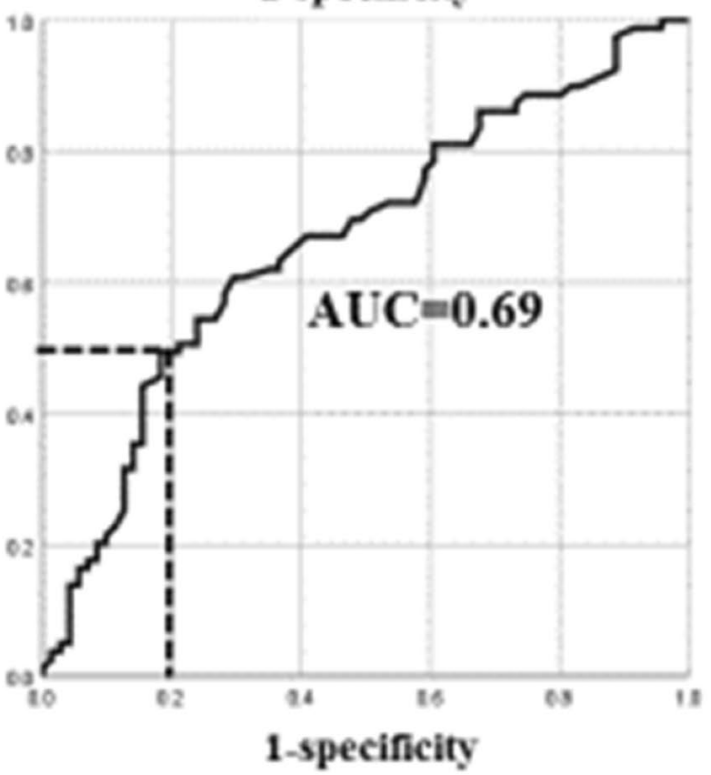

Fig. 7 ROC curve of clinical background and cephalometry for CPAP indication. a ROC curve of age for CPAP indication. $\mathbf{b}$ ROC curve of BMI for CPAP indication. $\mathbf{c}$ ROC curve of $\angle A N B$ for CPAP indication. d ROC curve of MP-H for CPAP indication. ROC curve receiver

Table 5 Number of subjects with and without risks of both BMI and MP-H

\begin{tabular}{lllc}
\hline Variables & $\begin{array}{l}\text { CPAP indica- } \\
\text { tion }\end{array}$ & $\begin{array}{l}\text { Not CPAP indi- } \\
\text { cation }\end{array}$ & Total \\
\hline $\begin{array}{l}\mathrm{BMI} \geq 26.0 \mathrm{~kg} / \mathrm{m}^{2} \text { and } \\
\mathrm{MP}-\mathrm{H} \geq 17.5 \mathrm{~mm}\end{array}$ & 52 & 7 & 59 \\
$\begin{array}{c}\mathrm{BMI}<26.0 \mathrm{~kg} / \mathrm{m}^{2} \text { and } \\
\mathrm{MP}-\mathrm{H}<17.5 \mathrm{~mm}\end{array}$ & 25 & 62 & 87 \\
\begin{tabular}{l} 
Total \\
\hline
\end{tabular} & 77 & 69 & 146 \\
\hline
\end{tabular}

$C P A P$ contentious positive airway pressure, $B M I$ body mass index, $M P-H$ distance from anterior-superior tip of hyoid bone to mandibular plane
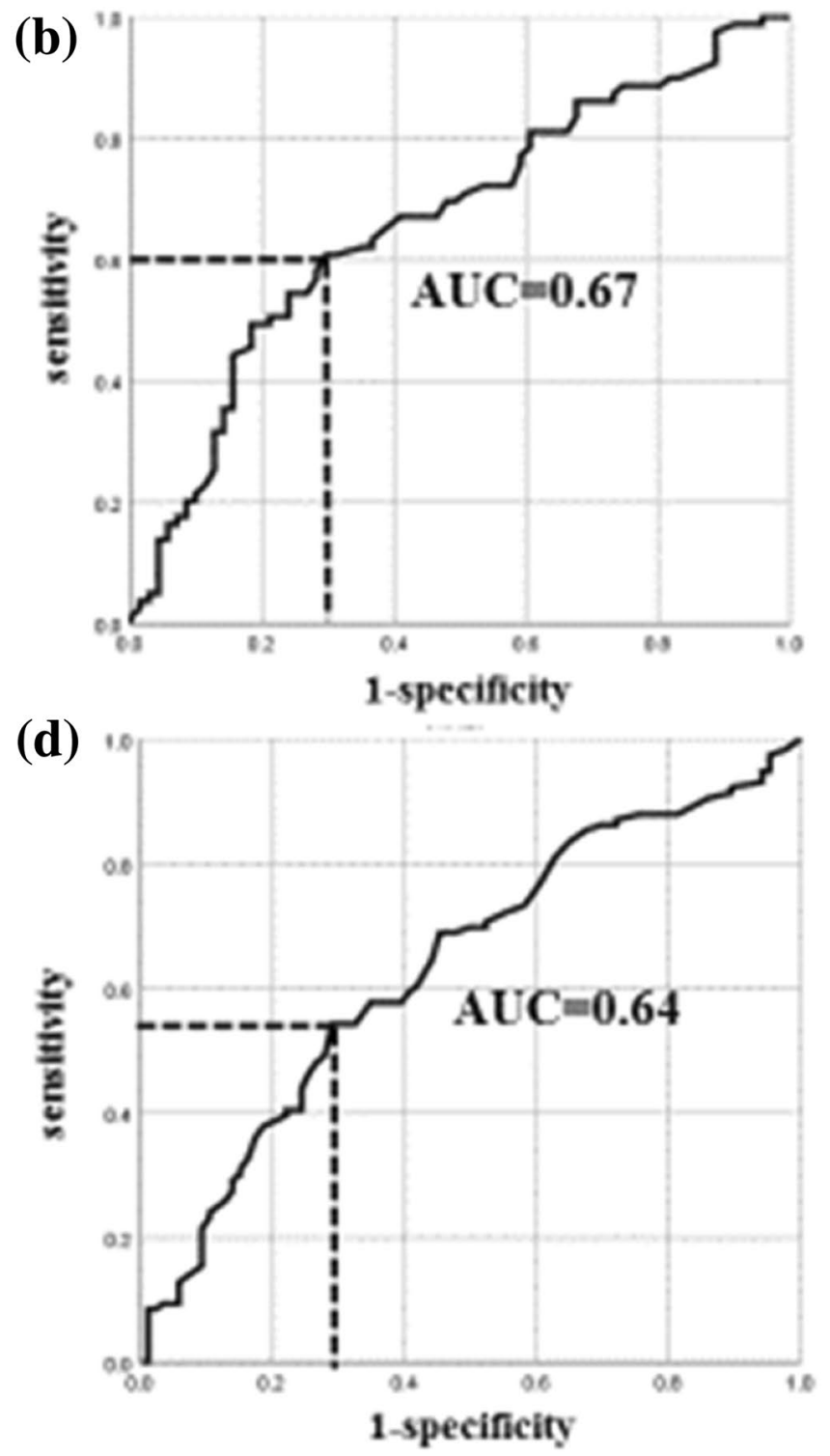

operating characteristic curve, $C P A P$ contentious positive airway pressure, $B M I$ body mass index, $\angle A N B$ lower jaw retraction, $M P-H$ distance from point anterior-superior tip of hyoid bone to mandibular plane

otherwise in a credit line to the material. If material is not included in the article's Creative Commons licence and your intended use is not permitted by statutory regulation or exceeds the permitted use, you will need to obtain permission directly from the copyright holder. To view a copy of this licence, visit http://creativecommons.org/licenses/by/4.0/.

\section{References}

1. Peppard PE, Young T, Barnet JH, Palta M, Hagen EW, Hla KM. Increased prevalence of sleep-disordered breathing in adults. Am J Epidemiol. 2013;177:1006-14. 
2. Miglis MG. Autonomic dysfunction in primary sleep disorders. Sleep Med. 2016;19:40-9.

3. Marin JM, Carrizo SJ, Vicente E, Agusti AGN. Long-term cardiovascular outcomes in men with obstructive sleep apnoea with or without treatment with continuous positive airway pressure: an observation study. Lancet. 2005;365:1046-53.

4. Flemons WW, Littner MR, Rowley JA, Gay P, Anderson WM, Hudgel DW, McEvoy RD, Loube D. Home diagnosis of sleep apnea: a systematic review of the literature. Chest. 2003;124:1543-79.

5. Russell T, Duntley S. Sleep disordered breathing in the elderly. Am J Med. 2011;124:1123-6.

6. Young T, Shahar E, Nieto FJ, Redline S, Newman AB, Gottlieb DJ, Walsleben JA, Finn L, Enright P, Samet JM, Sleep Health Study Research Group. Predictors of sleep-disordered breathing in community-dwelling adults: the sleep heart health study. Arch Intern Med. 2002;162:893-900.

7. Young T, Palta M, Dempsey J, Skatrud J, Weber S, Badr S. The occurrence of sleep-disordered breathing among middle-aged adluts. N Engl J Med. 1993;328:1230-5.

8. Redline S, Kump K, Tishler PV, Browner I, Ferrette V. Gender differences in sleep disordered breathing in a community-based sample. Am J Respir Crit Care Med. 1994;149:722-6.

9. Bixler EO, Vgontzas AN, Lin HM, Have TT, Rein J, Vela-Bueno A, Kales A. Prevalence of sleep-disordered breathing in women: effects of gender. Am J Respir Crit Care Med. 2001;163:608-13.

10. Cistulli PA, Grunstein RR, Sullivan CE. Effects of testosterone on upper airway collapsibility during during sleep. Am J Respir Crit Care Med. 1994;149:530-2.

11. Young T, Finn L, Austin D, Peterson A. Menopausal status and sleep-disordered breathing in the Wisconsin Sleep Cohort study. Am J Respir Crit Care Med. 2002;167:1181-5.

12. Murray WJ. Daytime sleepiness, snoring and obstructive sleep apnea: the Epworth Sleepiness Scale. Chest. 1993;103:30-6.

13. Sareli AE, Cantor CR, Williams NN, Korus G, Raper SE, Pien G, Hurley S, Maislin G, Schwab RJ. Obstructive sleep apnea in patients undergoing bariatric surgery-a tertiary center experience. Obes Surg. 2011;21:316-27.

14. Peppard PE, Young T, Palta M, Dempsey J, Skatrud J. Longitudinal study of moderate weight change and sleep-disordered breathing. JAMA. 2000;284:3015-21.

15. Riley R, Guilleminault C, Herran J, Powell N. Cephalometric analyses and flow-volume loops in obstructive sleep apnea patients. Sleep. 1983;6:303-11.

16. Gungor AY, Turkkahraman H, Yilmaz HH, Yariktas M. Cephalometric comparison of obstructive sleep apnea patients and healthy control. Eur J Dent. 2013;7:48-54.

17. Kubota Y, Nakayama H, Takada T, Matsuyama N, Sakaki K, Yoshizawa M, Nakamata M, Satoh M, Akazawa K, Suzuki E, Gejyo F. Facial axis angle as a risk factor for obstructive sleep apnea. Intern Med. 2005;44:805-10.

18. Pae EK, Lowe AA, Sasaki K, Price C, Tsuchiya M, Fleetham JA. A cephalometric and electromyographic study of upper airway structures in the upright and supine positions. Am J Orthod Dentofac Orthop. 1994;106:52-9.

19. Kikuchi M, Higurashi N, Miyazaki S, Itasaka Y. Facial patterns of obstructive sleep apnea patients using Ricketts method. Psychiatry Clin Neurosci. 2000;54:336-7.
20. Szendroedi J, Roben M. Ectopic lipids and organ function. Curr Opin Lipidol. 2009;20:50-6.

21. Sakakibara H, Tong M, Matsushita M, Hirata Y, Konishi S, Suetsugu S. Cephalometric abnormalities in non-obese and obese patients with obstructive sleep apnoea. Eur Respir J. 1999;13:403-10.

22. Fukuhara S, Takegami M, Suzukamo Y, Chin K, Inoue Y, Kadotani H, Oka Y, Noguchi H, Wakita T, Namikawa T, Nakamura T, Mishima M, Murray WJ. Japanese Edition the Epworth Sleepiness Scale (JESS)-Major differences and revisions from many 'Japanese versions' that have been used so far. Ann Jpn Respir Soc. 2006;44:896-8 (in Japanese).

23. American Academy of Sleep Medicine. International classification of sleep disorders. 3rd ed. Darien: American Academy of Sleep Medicine; 2014.

24. Kitamura T, Sakabe A, Ueda N, Shiomori T, Udaka T, Ohbuchi T, Suzuki H. Usefulness of cephalometry and pharyngeal finding in the primary diagnosis of obstructive sleep apnea syndrome. Nippon Jibiinkoka Gakkai Kaiho. 2008;111:695-700.

25. Esaki K. Morphological analysis by lateral cephalography of sleep apnea syndrome in 53 patients. Kurume Med J. 1995;42:231-40.

26. Yao M, Tachibana N, Okura M, Tanigawa T, Yamagishi K, Ikeda A, Shimamoto T, Iso H. Relationships of craniofacial morphology and body mass index with sleep-disordered breathing in Japanese men. Laryngoscope. 2004;114:1838-42.

27. Ancoli-Israel S, Krinpke DF, Klauber MR, Mason WJ, Fell R, Kaplan O. Sleep-disordered breathing in community-dwelling elderly. Sleep. 1991;14:486-95.

28. Philip P, Dealberto MJ, Dartigues JF, Guilleminault C, Bioulac B. Prevalence and correlates of nocturnal desaturation in a sample of elderly people. J Sleep Res. 1997;6:264-71.

29. Gottlied DJ, Whitney CW, Bonekat WH, Iber C, James GD, Lebowitz M, Nieto FJ, Rosenberg CE. Relation of sleepiness to respiratory disturbance index: the sleep heart health study. Am J Respir Crit Care Med. 1999;159:502-7.

30. Leticia B, Carolina MC, Samantha RFDB, Oscimar BS, Jose ELD. Evaluation of Epworth Sleepiness Scale in patients with obstructive sleep apnea hypopnea syndrome. Rev Bras Otorrinolaringol. 2004;70:752-6.

31. Douglas NJ. The obstructive sleep apnea/hypopnea syndrome. Pract Neurol. 2003;3:22-9.

32. Kaneita Y, Ohida T, Uchiyama MM, Takemura S, Kawahara K, Yokoyama E, Miyake T, Hirano S, Suzuki K, Yagi Y, Akiyo Kaneko A, Tsutsumi T, Akashiba T. Excessive daytime sleepiness among the Japanese general population. J Epidemiol. 2005; $15: 1-8$.

33. Tarasiuk A, Greenberg-Dotan S, Simon T, Tal A, Oksenberg A, Reuveni H. Low socioeconomic status is a risk factor for cardiovascular disease among adult obstructive sleep apnea syndrome patients requiring treatment. Chest. 2006;130:766-73.

34. Hoffstein V. Apnea and snoring: state of the art and future directions. Acta Otorhinolaryngol Belg. 2002;56:205-36.

Publisher's Note Springer Nature remains neutral with regard to jurisdictional claims in published maps and institutional affiliations. 